CRYSTALLOGRAPHIC COMMUNICATIONS

ISSN 2056-9890

Received 17 March 2020

Accepted 23 March 2020

Edited by S. Parkin, University of Kentucky, USA

Keywords: crystal structure; tryptamines; indoles; hydrogen bonding.

CCDC references: 1992279; 1992278

Supporting information: this article has supporting information at journals.iucr.org/e

\section{Norpsilocin: freebase and fumarate salt}

\author{
Andrew R. Chadeayne, ${ }^{\mathrm{a} *}$ Duyen N. K. Pham, ${ }^{\mathrm{b}}$ James A. Golen ${ }^{\mathrm{b}}$ and David R. \\ Manke ${ }^{b}$

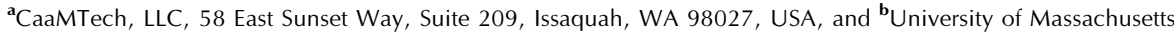 \\ Dartmouth, 285 Old Westport Road, North Dartmouth, MA 02747, USA. *Correspondence e-mail: andrew@caam.tech
}

The solid-state structures of the naturally occurring psychoactive tryptamine norpsilocin \{4-hydroxy- $N$-methyltryptamine (4-HO-NMT); systematic name: 3-[2-(methylamino)ethyl]- $1 H$-indol-4-ol $\}, \mathrm{C}_{11} \mathrm{H}_{14} \mathrm{~N}_{2} \mathrm{O}$, and its fumarate salt (4-hydroxy- $N$-methyltryptammonium fumarate; systematic name: bis $\{[2-(4-h y-$ droxy-1H-indol-3-yl)ethyl]methylazanium\} but-2-enedioate), $\mathrm{C}_{11} \mathrm{H}_{15} \mathrm{~N}_{2} \mathrm{O}^{+}$.$0.5 \mathrm{C}_{4} \mathrm{H}_{2} \mathrm{O}_{4}{ }^{2-}$, are reported. The freebase of 4-HO-NMT has a single molecule in the asymmetric unit joined together by $\mathrm{N}-\mathrm{H} \cdots \mathrm{O}$ and $\mathrm{O}-\mathrm{H} \cdots \mathrm{O}$ hydrogen bonds in a two-dimensional network parallel to the (100) plane. The ethylamine arm of the tryptamine is modeled as a two-component disorder with a 0.895 (3) to 0.105 (3) occupancy ratio. The fumarate salt of 4-HO-NMT crystallizes with a tryptammonium cation and one half of a fumarate dianion in the asymmetric unit. The ions are joined together by $\mathrm{N}-\mathrm{H} \cdots \mathrm{O}$ and $\mathrm{O}-\mathrm{H} \cdots \mathrm{O}$ hydrogen bonds to form a three-dimensional framework, as well as $\pi-\pi$ stacking between the sixmembered rings of inversion-related indoles (symmetry operation: $2-x, 1-y$, $2-z)$.

\section{Chemical context}

Psychoactive tryptamines, particularly psilocybin and psilocin, have recently garnered a great deal of interest because of their potential to treat disorders including anxiety, addiction, and depression (Johnson \& Griffiths, 2017; Carhart-Harris \& Goodwin, 2017). Of note, psilocybin was recently granted the 'breakthrough therapy' designation by the US Food and Drug Administration (Feltman, 2019). To this point, the focus of research on psychedelics in therapy has largely been on psilocybin and psilocin. Despite this focus, there are more than 200 species of 'magic mushrooms' containing many different psychoactive tryptamines and combinations of the same (Stamets, 1996).

The clinical effects observed for extracts of 'magic mushrooms' differ from those observed for pure psilocybin (Zhuk, et al. 2015). This indicates that the minor components of 'magic mushrooms' have psychoactive properties that are important, or that they work in conjunction with psilocybin as part of an entourage effect (Russo, 2011). To have a better understanding of 'magic mushroom' pharmacology, it is necessary to understand the properties of the minor active components. This could lead to formulations that maximize the desired activity while minimizing negative effects, optimizing the clinical experience.

Baeocystin, the monomethyl analog of psilocybin, is the second most abundant naturally occurring tryptamine found in 'magic mushrooms'. It was first isolated from the mushroom 
Psilocybe baeocystis in 1968 (Leung \& Paul, 1968), and subsequently identified in other species, approaching one third of the total tryptamine concentration. Like psilocybin, baeocystin acts as a prodrug when consumed by humans, undergoing rapid hydrolysis of the phosphate ester to afford its active metabolite - the 4-hydroxy analog.

The prodrug psilocybin hydrolyses to the active 4-hydroxy$\mathrm{N}, \mathrm{N}$-dimethyltryptamine (4-HO-DMT), aka psilocin, and the prodrug baeocystin hydrolyses to the active 4-hydroxy- $\mathrm{N}$ methyltryptamine (4-HO-NMT), aka norpsilocin. Norpsilocin was first identified as a natural product of 'magic mushrooms' in 2017, and isolated as an amorphous, colorless solid (Lenz et al., 2017). In 2020, norpsilocin was synthesized and isolated as a white solid in $98 \%$ purity. When tested as an agonist at the human seratonin $2 \mathrm{a}$ receptor, synthetic norpsilocin was as potent, if not more so, compared to psilocin (Sherwood et al., 2020).

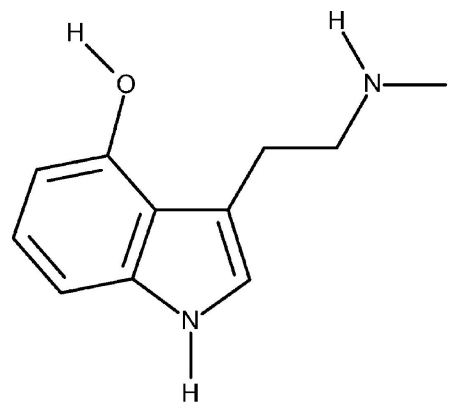

(I)

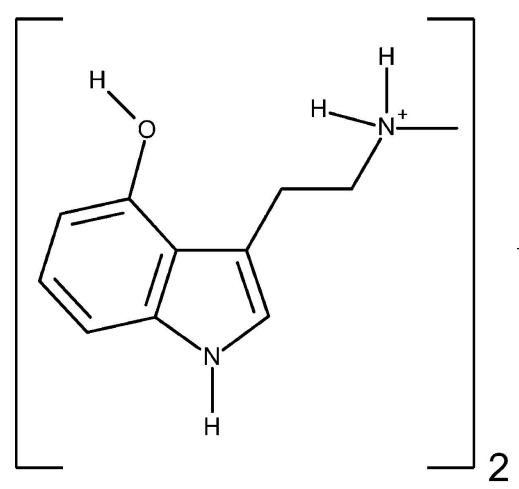<smiles>O=C([O-])CCC(=O)[O-]</smiles>

(II)

Despite rapidly growing evidence supporting psilocin/ psilocybin's potential for treating mood disorders, very little work has been done to investigate the properties of other structurally similar compounds found in magic mushrooms, e.g. norpsilocin/baeocystin. Although these compounds have substantial potential as drug candidates, they have undergone limited investigation because of their lack of availability in pure form and the difficulty of their purification. Crystalline solids are the most convenient and reliable chemical forms for studying, handling, and administering pure compounds. There was an unmet need for the structural characterization of norpsilocin, which is important in examining the structureactivity relationship of the psychedelic tryptamine. Herein, we report the first crystal structure of norpsilocin (I), and the first salt of norpsilocin (II) and its solid-state structure.

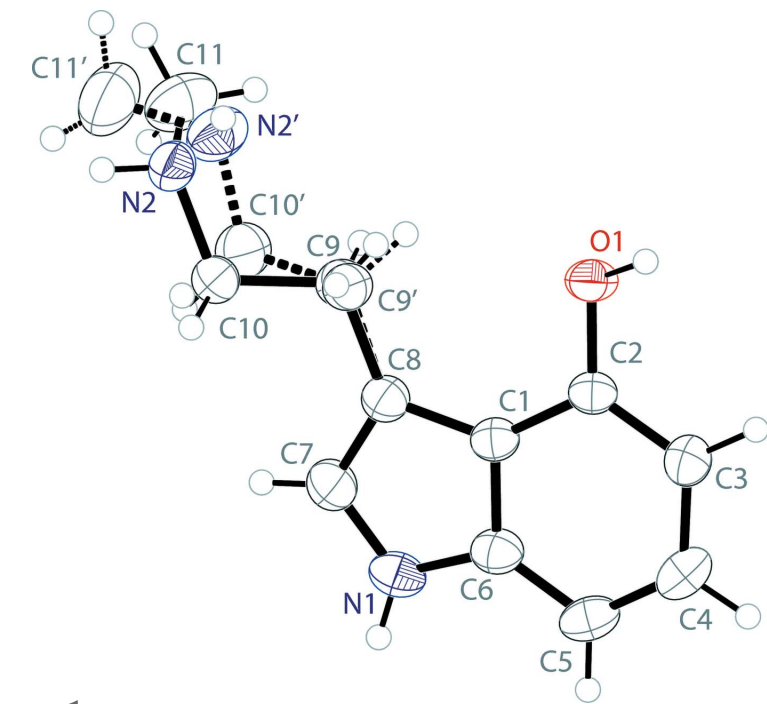

Figure 1

The molecular structure of 4-hydroxy- $N$-methyltryptamine, showing the atom labeling. Displacement ellipsoids are drawn at the $50 \%$ probability level. Dashed bonds indicate the minor occupancy disordered component in the structure.

\section{Structural commentary}

The molecular structure of the freebase of norpsilocin, 4-HONMT, is shown in Fig. 1. The asymmetric unit contains one full 4-hydroxy- $N$-methyltryptamine $\left(\mathrm{C}_{11} \mathrm{H}_{14} \mathrm{~N}_{2} \mathrm{O}\right)$ molecule. The ethylamine arm $(\mathrm{C} 9-\mathrm{C} 10-\mathrm{N} 2-\mathrm{C} 11)$ of the tryptamine is modeled as a two-component disorder with a 0.895 (3) to 0.105 (3) occupancy ratio. The rest of the discussion is restricted to the major component. The indole ring system of the tryptamine is near planar with an r.m.s. deviation from planarity of $0.015 \AA$. The ethylamine arm of the tryptamine is slightly turned, with a $\mathrm{C} 7-\mathrm{C} 8-\mathrm{C} 9-\mathrm{C} 10$ torsion angle of $29.3(3)^{\circ}$. The $\mathrm{C} 10-\mathrm{N} 2-\mathrm{C} 11$ angle about the amine nitrogen is $113.51(15)^{\circ}$.

The molecular structure of the fumarate salt of norpsilocin is shown in Fig. 2. The asymmetric unit contains one full 4-hydroxy- $N$-methyltryptammonium $\left(\mathrm{C}_{11} \mathrm{H}_{15} \mathrm{~N}_{2} \mathrm{O}^{+}\right)$cation and one half of a fumarate $\left(\mathrm{C}_{4} \mathrm{H}_{2} \mathrm{O}_{4}{ }^{2-}\right)$ dianion, with the other half generated by inversion. The indole ring system of the

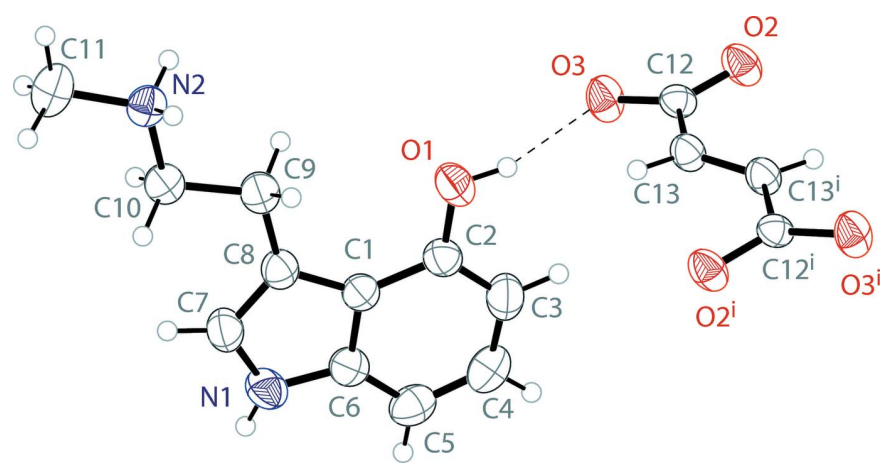

Figure 2

The molecular structure of bis(4-hydroxy- $N$-methyltryptammonium)fumarate, showing the atom labeling. Displacement ellipsoids are drawn at the $50 \%$ probability level. Hydrogen bonds are shown as dashed lines. Symmetry code: (i) $1-x,-y, 2-z$. 
Table 1

Hydrogen-bond geometry $\left(\AA,^{\circ}\right)$ for (I).

\begin{tabular}{lllll}
\hline$D-\mathrm{H} \cdots A$ & $D-\mathrm{H}$ & $\mathrm{H} \cdots A$ & $D \cdots A$ & $D-\mathrm{H} \cdots A$ \\
\hline $\mathrm{O} 1-\mathrm{H} 1 \cdots \mathrm{N} 2^{\mathrm{i}}$ & $0.86(1)$ & $1.80(1)$ & $2.6501(16)$ & $169(2)$ \\
$\mathrm{N} 1-\mathrm{H} 1 A \cdots \mathrm{O} 1^{\mathrm{ii}}$ & $0.88(1)$ & $2.04(1)$ & $2.9092(15)$ & $175(2)$ \\
\hline
\end{tabular}

Symmetry codes: (i) $-x+1,-y+1,-z+1$; (ii) $x,-y+\frac{3}{2}, z-\frac{1}{2}$.

tryptamine is near planar with an r.m.s. deviation from planarity of $0.009 \AA$. Unlike the freebase, the ethyl ammonium arm resides in the same plane as the indole. The planarity of all of the non-hydrogen atoms of the tryptamine is demonstrated with an r.m.s. deviation from planarity of only $0.043 \AA$. The $\mathrm{C} 10-\mathrm{N} 2-\mathrm{C} 11$ angle about the ammonium nitrogen is $114.20(14)^{\circ}$. The fumarate itself is also near planar, with an r.m.s. deviation from planarity of $0.050 \AA$. The carboxylate unit of the fumarate is delocalized, with $\mathrm{C}-\mathrm{O}$ distances of 1.2488 (18) and 1.2553 (18) A.

\section{Supramolecular features}

The tryptamine molecules of the freebase of norpsilocin are held in an infinite two-dimensional network parallel to the (100) plane through a series of $\mathrm{N}-\mathrm{H} \cdots \mathrm{O}$ and $\mathrm{O}-\mathrm{H} \cdots \mathrm{N}$ hydrogen bonds (Table 1 ). The phenol $\mathrm{O}-\mathrm{H}$ hydrogen bonds with the nitrogen of the methylamine of an inversion-related tryptamine molecule (symmetry operation: $-x+1,-y+1$,

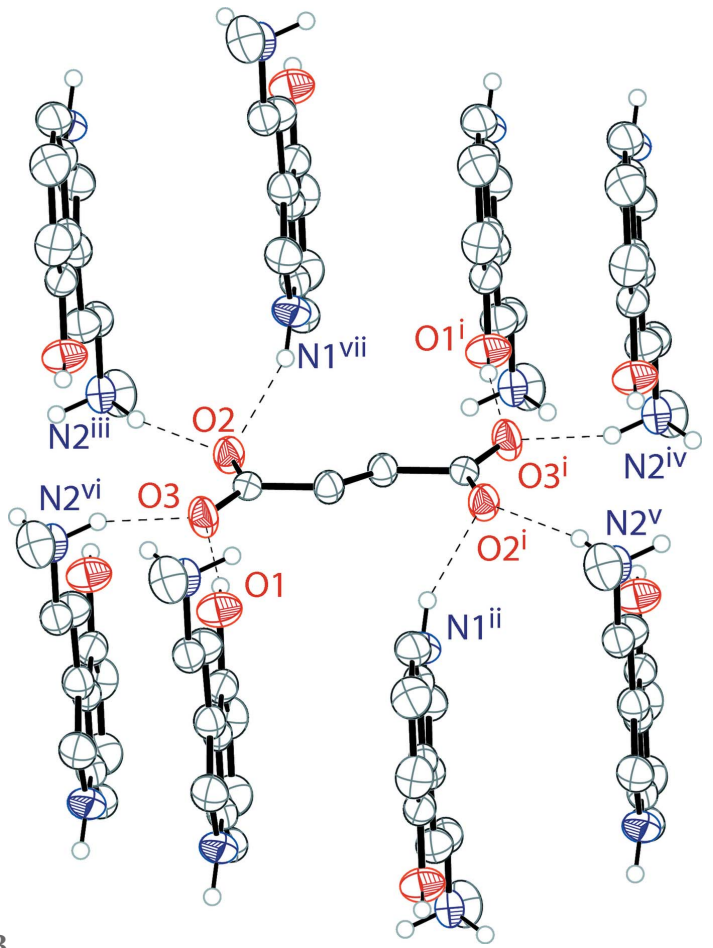

Figure 3

The hydrogen bonding (Table 2) of a fumarate ion in the structure of bis(4-hydroxy- $N$-methyltryptammonium)fumarate, with hydrogen bonds shown as dashed lines. Displacement ellipsoids are drawn at the $50 \%$ probability level. Hydrogen atoms not involved in hydrogen bonding are omitted for clarity. Symmetry codes: (i) $1-x,-y, 2-z$; (ii) $2-x, 1-y$, $2-z$; (iii) $1-x,-y, 1-z$; (iv) $2-x,-y, 2-z$; (v) $x, y, 1+z$; (vi) $-1+x$, $y, z ;($ vii) $-1+x,-1+y, z$.
Table 2

Hydrogen-bond geometry $\left(\AA,^{\circ}\right)$ for (II).

\begin{tabular}{lllll}
\hline$D-\mathrm{H} \cdots A$ & $D-\mathrm{H}$ & $\mathrm{H} \cdots A$ & $D \cdots A$ & $D-\mathrm{H} \cdots A$ \\
\hline $\mathrm{O} 1-\mathrm{H} 1 \cdots \mathrm{O} 3$ & $0.87(1)$ & $1.89(1)$ & $2.7399(16)$ & $163(2)$ \\
$\mathrm{N} 1-\mathrm{H} 1 A \cdots \mathrm{O} 2^{\mathrm{i}}$ & $0.86(1)$ & $2.07(1)$ & $2.8854(18)$ & $157(2)$ \\
$\mathrm{N}^{\mathrm{ii}}-\mathrm{H} 2 A \cdots 3^{\mathrm{i}}$ & $0.89(1)$ & $1.90(1)$ & $2.7349(18)$ & $155(2)$ \\
$\mathrm{N} 2-\mathrm{H} 2 B \cdots \mathrm{O} 2^{\mathrm{iii}}$ & $0.89(1)$ & $1.91(1)$ & $2.7715(19)$ & $164(2)$ \\
\hline
\end{tabular}

Symmetry codes: (i) $x+1, y+1, z$; (ii) $x+1, y, z$; (iii) $-x+1,-y,-z+1$.

$-z+1)$ to form a dimer. The indole $\mathrm{N}-\mathrm{H}$ shows an intermolecular hydrogen bond with the phenol oxygen of another tryptamine molecule (symmetry operation: $x,-y+\frac{3}{2}, z-\frac{1}{2}$ ), joining the dimers into two-dimensional sheets. The packing of 4-HO-NMT is shown in Fig. $4 a$.

(a)

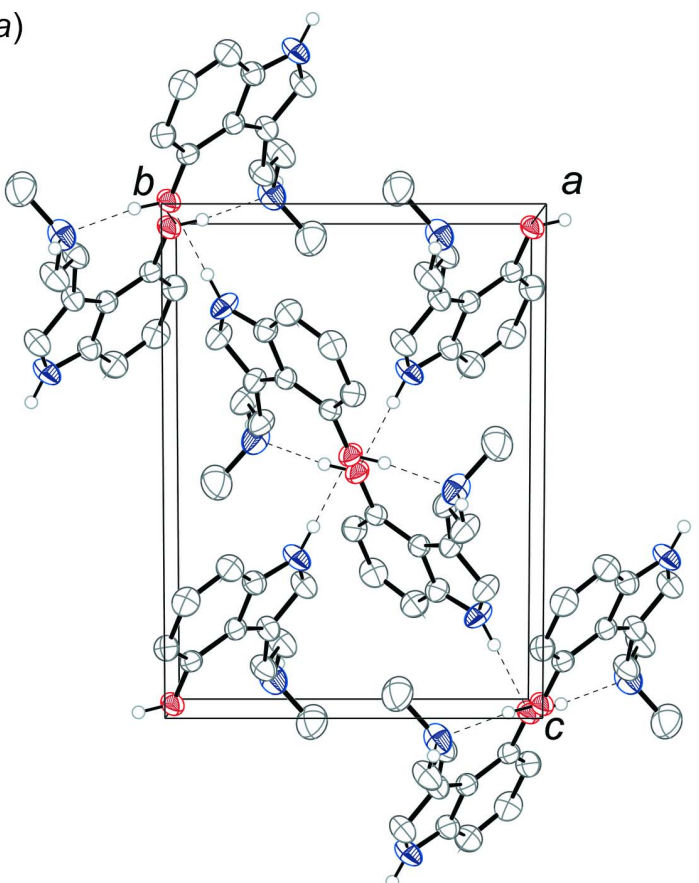

(b)

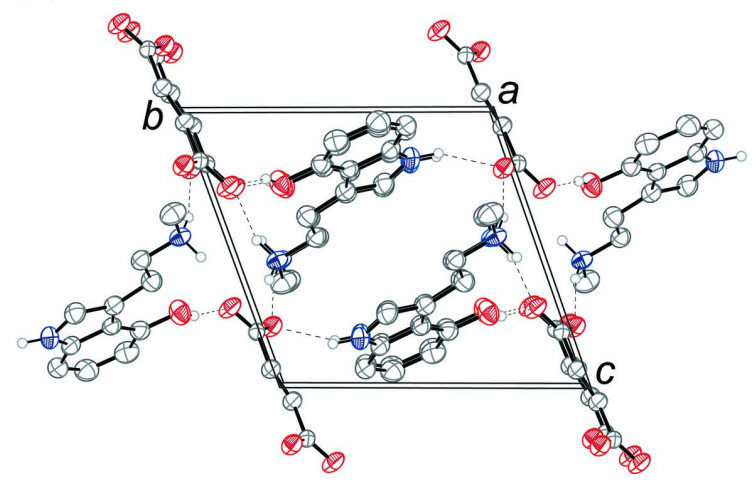

Figure 4

The crystal packing of (a) 4-HO-NMT, and of $(b)$ bis(4-HO-NMT) fumarate, both shown along the $a$ axis. The hydrogen bonds (Tables 1 and 2) are shown as dashed lines. Displacement ellipsoids are drawn at the $50 \%$ probability level. Hydrogen atoms not involved in hydrogen bonding are omitted for clarity. For ( $a$ ) only one component of the disorder is shown. 
Table 3

Experimental details.

(I)

Crystal data

Chemical formula

$M_{\mathrm{r}}$

Crystal system, space group

Temperature (K)

$a, b, c(\AA)$

$\alpha, \beta, \gamma\left({ }^{\circ}\right)$

$V\left(\AA^{3}\right)$

$Z$

Radiation type

$\mu\left(\mathrm{mm}^{-1}\right)$

Crystal size (mm)

Data collection

Diffractometer

Absorption correction

$T_{\min }, T_{\max }$

No. of measured, independent and

observed $[I>2 \sigma(I)]$ reflections

$R_{\text {int }}$

$(\sin \theta / \lambda)_{\max }\left(\AA^{-1}\right)$

Refinement

$R\left[F^{2}>2 \sigma\left(F^{2}\right)\right], w R\left(F^{2}\right), S$

No. of reflections

No. of parameters

No. of restraints

$\mathrm{H}$-atom treatment

$\Delta \rho_{\max }, \Delta \rho_{\min }\left(\mathrm{e} \AA^{-3}\right)$
(II)

\author{
$\mathrm{C}_{11} \mathrm{H}_{14} \mathrm{~N}_{2} \mathrm{O}$ \\ 190.24 \\ Monoclinic, $P 2_{1} / c$ \\ 297 \\ 9.4060 (16), 8.8436 (15), 12.144 (2) \\ $90,100.601$ (7), 90 \\ $993.0(3)$ \\ 4 \\ Mo $K \alpha$ \\ 0.08 \\ $0.35 \times 0.2 \times 0.1$
}

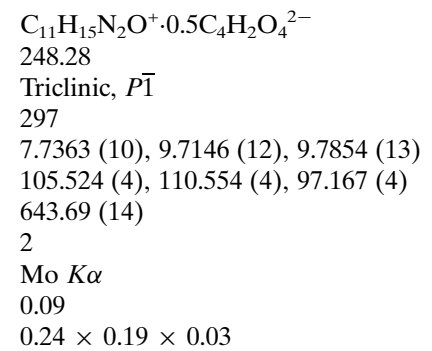

Bruker D8 Venture CMOS

Multi-scan (SADABS; Bruker, 2018)

$0.716,0.745$

$35681,1955,1687$

Bruker D8 Venture CMOS

Multi-scan (SADABS; Bruker, 2018)

$0.685,0.745$

$14395,2365,1774$

0.031

0.620

0.046

0.605

$0.038,0.105,1.09$
1955
171
105
$\mathrm{H}$ atoms treated by a mixture of independent
$\quad$ and constrained refinement
$0.20,-0.14$

$0.039,0.098,1.11$

2365

181

4

$\mathrm{H}$ atoms treated by a mixture of independent and constrained refinement

$0.15,-0.15$

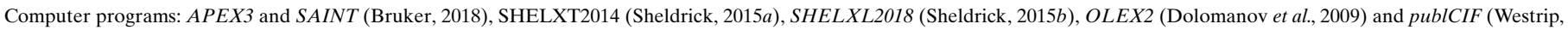
2010).

The tryptammonium cations and the fumarate dianions of the fumarate salt of norpsilocin are held together in an infinite three-dimensional framework through a series of $\mathrm{N}-\mathrm{H} \cdots \mathrm{O}$ and $\mathrm{O}-\mathrm{H} \cdots \mathrm{O}$ hydrogen bonds (Table 2). The indole $\mathrm{N}-\mathrm{H}$, methylammonium $\mathrm{N}-\mathrm{H}$, and phenol $\mathrm{O}-\mathrm{H}$ groups all hydrogen bond with the oxygen atoms of the fumarate dianion (Fig. 3). The six-membered rings of inversion-related indoles stack with parallel slipped $\pi-\pi$ interactions [intercentroid distance = 3.6465 (15) $\AA$, interplanar distance = 3.4781 (16) $\AA$, and slippage $=1.095(3) \AA]$. The packing of bis(4-HO-NMT) fumarate is shown in Fig. $4 b$.

\section{Database survey}

The most significant comparison to the structure of freebase norpsilocin is psilocin [CSD (Groom et al., 2016) refcode PSILIN: Petcher \& Weber, 1974). In the case of psilocin, the molecule dimerizes through $\mathrm{O}-\mathrm{H} \cdots \mathrm{N}$ hydrogen bonds, and does not form an extended network because of the lack of $\mathrm{N}-$ $\mathrm{H}$... O hydrogen bonds. The other free-base tryptamines whose structures are known include natural products such as psilocybin (PSILOC: Weber \& Petcher, 1974), DMT - N,Ndimethyltryptamine (DMTRYP: Falkenberg, 1972b) and bufotenine (BUFTEN: Falkenberg, 1972a), as well as synthetic tryptamines such as $N$-methyl- $N$-propyltryptamine (WOHYAW: Chadeayne, Golen \& Manke, 2019b).
The fumarate salt of norpsilocin crystallizes as a two-to-one tryptammonium-to-fumarate salt. This ratio has also been observed in salts of 4-acetoxy- $N, N$-dimethyltryptammonium (XOFDOO: Chadeayne, Golen \& Manke, 2019a), 4-hydroxy$N, N$-dipropyltryptammonium (CCDC 1962339: Chadeayne, Pham et al., 2019b), and 4-hydroxy- $N$-isopropyl- $N$-methyltryptammonium (CCDC 1987588: Chadeayne et al., 2020). One-to-one tryptammonium-to-hydrofumarate salts have been observed for 4-acetoxy- $N, N$-dimethyltryptammonium (HOCJUH: Chadeayne et al., 2019c), 4-hydroxy- $N$-isopropyl$N$-methyltryptammonium and $N$-isopropyl- $N$-methyltyptammonium (RONSUL and RONSOF: Chadeayne, Pham et al., 2019a).

\section{Synthesis and crystallization}

Single crystals suitable for X-ray analysis were obtained from the slow evaporation of an acetone solution of a commercial sample of 4-hydroxy- $N$-methyltryptamine (Angene).

The fumarate salt was synthesized starting with $101 \mathrm{mg}$ of 4-hydroxy- $N$-methyltryptamine, which was dissolved in $10 \mathrm{~mL}$ of methanol. $62 \mathrm{mg}$ of fumaric acid was added to the solution and it was stirred overnight under reflux. Solvent was removed in vacuo to yield a dark-blue powder. The powder was triturated with diethyl ether and then recrystallized in acetone to yield colorless crystals suitable for X-ray analysis. ${ }^{1} \mathrm{H}$ NMR (400 MHz, $\left.\mathrm{D}_{2} \mathrm{O}\right): \delta 7.12(s, 1 \mathrm{H}, \operatorname{ArH}), 7.10-7.07(m, 2 \mathrm{H}$, 
$\operatorname{Ar} H), 6.66(s, 2 \mathrm{H}, \mathrm{CH}), 6.56(d d, J=5.5,2.8 \mathrm{~Hz}, 1 \mathrm{H}, \mathrm{Ar} H)$, $3.41\left(t, J=6.8 \mathrm{~Hz}, 2 \mathrm{H}, \mathrm{CH}_{2}\right), 3.26\left(t, J=6.8 \mathrm{~Hz}, \mathrm{CH}_{2}\right), 2.70(s$, $\left.3 \mathrm{H}, \mathrm{CH}_{3}\right) ;{ }^{13} \mathrm{C} \mathrm{NMR}\left(100 \mathrm{MHz}, \mathrm{D}_{2} \mathrm{O}\right): \delta 171.0(\mathrm{COOH}), 149.7$ $(\mathrm{ArC}), 138.5(\mathrm{ArC}), 134.2(\mathrm{CH}), 123.0(\mathrm{ArC}), 122.8(\mathrm{ArC})$, 115.6 ( $\mathrm{ArC}), 108.4(\mathrm{ArC}), 104.2(\mathrm{ArC}), 103.4(\mathrm{ArC}), 50.3$ $\left(\mathrm{CH}_{2}\right), 32.4\left(\mathrm{CH}_{2}\right), 22.7\left(\mathrm{CH}_{3}\right)$.

\section{Refinement}

Crystal data, data collection and structure refinement details are summarized in Table 3. Hydrogen atoms $\mathrm{H} 1, \mathrm{H} 1 A$, and $\mathrm{H} 2$ were found from a difference-Fourier map and were refined isotropically, using DFIX restraints with $\mathrm{N}-\mathrm{H}$ distances of 0.87 (1) $\AA$ and an $\mathrm{O}-\mathrm{H}$ distance of 0.88 (1) $\AA$. Isotropic displacement parameters were set to $1.2 U_{\text {eq }}$ of the parent nitrogen atom and $1.5 U_{\text {eq }}$ of the parent oxygen atom. All other hydrogen atoms were placed in calculated positions $(\mathrm{C}-\mathrm{H}=$ 0.93-0.97 $\AA$ ). Isotropic displacement parameters were set to $1.2 U_{\text {eq }}(\mathrm{C})$ or $1.5 U_{\text {eq }}(\mathrm{C}-$ methyl $)$.

\section{Acknowledgements}

Financial statements and conflict of interest: This study was funded by CaaMTech, Inc. ARC reports an ownership interest in CaaMTech, Inc., which owns US and worldwide patent applications, covering new tryptamine compounds, compositions, formulations, novel crystalline forms, and methods of making and using the same.

\section{Funding information}

Funding for this research was provided by: National Science Foundation, Directorate for Mathematical and Physical Sciences (grant No. CHE-1429086).

\section{References}

Bruker (2018). APEX3, SAINT, and SADABS. Bruker AXS Inc., Madison, Wisconsin, USA.
Carhart-Harris, R. L. \& Goodwin, G. M. (2017). Neuropsychopharmacology, 42, 2105-2113.

Chadeayne, A. R., Golen, J. A. \& Manke, D. R. (2019a). Acta Cryst. E75, 900-902.

Chadeayne, A. R., Golen, J. A. \& Manke, D. R. (2019b). IUCrData, 4, x190962.

Chadeayne, A. R., Golen, J. A. \& Manke, D. R. (2019c). Psychedelic Science Review. https://psychedelicreview.com/the-crystalstructure-of-4-aco-dmt-fumarate/

Chadeayne, A. R., Pham, D. N. K., Golen, J. A. \& Manke, D. R. (2019a). Acta Cryst. E75, 1316-1320.

Chadeayne, A. R., Pham, D. N. K., Golen, J. A. \& Manke, D. R. (2019b). IUCrData, 4, x191469.

Chadeayne, A. R., Pham, D. N. K., Golen, J. A. \& Manke, D. R. (2020). Acta Cryst. E76, 514-517.

Dolomanov, O. V., Bourhis, L. J., Gildea, R. J., Howard, J. A. K. \& Puschmann, H. (2009). J. Appl. Cryst. 42, 339-341.

Falkenberg, G. (1972a). Acta Cryst. B28, 3075-3083.

Falkenberg, G. (1972b). Acta Cryst. B28, 3219-3228.

Feltman, R. (2019). Popular Science. https://popsci. com/story/health/ psilocybin-magic-mushroom-fda-breakthrough-depression/

Groom, C. R., Bruno, I. J., Lightfoot, M. P. \& Ward, S. C. (2016). Acta Cryst. B72, 171-179.

Johnson, M. W. \& Griffiths, R. R. (2017). Neurotherapeutics 14, 734740.

Lenz, C., Wick, J. \& Hoffmeister, D. (2017). J. Nat. Prod. 80, 28352838.

Leung, A. Y. \& Paul, A. G. (1968). J. Pharm. Sci. 57, 1667-1671.

Petcher, T. J. \& Weber, H. P. (1974). J. Chem. Soc. Perkin Trans. 2, pp. 946-948.

Russo, E. B. (2011). Br. J. Pharmacol. 163, 1344-1364.

Sheldrick, G. M. (2015a). Acta Cryst. A71, 3-8.

Sheldrick, G. M. (2015b). Acta Cryst. C71, 3-8.

Sherwood, A. M., Halberstadt, A. L., Klein, A. K., McCorvy, J. D., Kaylo, K. W., Kargbo, R. B. \& Meisenheimer, P. (2020). J. Nat. Prod. 83, 461-467.

Stamets, P. (1996). Psilocybin mushrooms of the world: An identification guide. Berkeley, CA: Ten Speed Press.

Weber, H. P. \& Petcher, T. J. (1974). J. Chem. Soc. Perkin Trans. 2, pp. 942-946.

Westrip, S. P. (2010). J. Appl. Cryst. 43, 920-925.

Zhuk, O., Jasicka-Misiak, I., Poliwoda, A., Kazakova, A., Godovan, V. V., Halama, M. \& Wieczorek, P. (2015). Toxins, 7, 10181029. 


\section{supporting information}

Acta Cryst. (2020). E76, 589-593 [https://doi.org/10.1107/S2056989020004077]

Norpsilocin: freebase and fumarate salt

Andrew R. Chadeayne, Duyen N. K. Pham, James A. Golen and David R. Manke

Computing details

For both structures, data collection: APEX3 (Bruker, 2018); cell refinement: SAINT (Bruker, 2018); data reduction: SAINT (Bruker, 2018); program(s) used to solve structure: SHELXT2014 (Sheldrick, 2015a); program(s) used to refine structure: SHELXL2018 (Sheldrick, 2015b); molecular graphics: OLEX2 (Dolomanov et al., 2009); software used to prepare material for publication: publCIF (Westrip, 2010).

3-[2-(Methylamino)ethyl]-1H-indol-4-ol (I)

Crystal data

$\mathrm{C}_{11} \mathrm{H}_{14} \mathrm{~N}_{2} \mathrm{O}$

$M_{r}=190.24$

Monoclinic, $P 22_{1} / c$

$a=9.4060(16) \AA$

$b=8.8436(15) \AA$

$c=12.144(2) \AA$

$\beta=100.601(7)^{\circ}$

$V=993.0(3) \AA^{3}$

$Z=4$

Data collection

Bruker D8 Venture CMOS diffractometer

$\varphi$ and $\omega$ scans

Absorption correction: multi-scan

(SADABS; Bruker, 2018)

$T_{\min }=0.716, T_{\max }=0.745$

35681 measured reflections

\section{Refinement}

Refinement on $F^{2}$

Least-squares matrix: full

$R\left[F^{2}>2 \sigma\left(F^{2}\right)\right]=0.038$

$w R\left(F^{2}\right)=0.105$

$S=1.09$

1955 reflections

171 parameters

105 restraints
$F(000)=408$

$D_{\mathrm{x}}=1.273 \mathrm{Mg} \mathrm{m}^{-3}$

Mo $K \alpha$ radiation, $\lambda=0.71073 \AA$

Cell parameters from 9944 reflections

$\theta=2.9-26.0^{\circ}$

$\mu=0.08 \mathrm{~mm}^{-1}$

$T=297 \mathrm{~K}$

BLOCK, colourless

$0.35 \times 0.2 \times 0.1 \mathrm{~mm}$

1955 independent reflections

1687 reflections with $I>2 \sigma(I)$

$R_{\text {int }}=0.031$

$\theta_{\max }=26.1^{\circ}, \theta_{\min }=3.2^{\circ}$

$h=-11 \rightarrow 11$

$k=-10 \rightarrow 10$

$l=-14 \rightarrow 15$

Hydrogen site location: mixed

$\mathrm{H}$ atoms treated by a mixture of independent and constrained refinement

$w=1 /\left[\sigma^{2}\left(F_{\mathrm{o}}^{2}\right)+(0.050 P)^{2}+0.2586 P\right]$

where $P=\left(F_{\mathrm{o}}^{2}+2 F_{\mathrm{c}}{ }^{2}\right) / 3$

$(\Delta / \sigma)_{\max }<0.001$

$\Delta \rho_{\max }=0.20 \mathrm{e} \AA^{-3}$

$\Delta \rho_{\min }=-0.14$ e $\AA^{-3}$ 


\section{Special details}

Geometry. All esds (except the esd in the dihedral angle between two 1.s. planes) are estimated using the full covariance matrix. The cell esds are taken into account individually in the estimation of esds in distances, angles and torsion angles; correlations between esds in cell parameters are only used when they are defined by crystal symmetry. An approximate (isotropic) treatment of cell esds is used for estimating esds involving l.s. planes.

Fractional atomic coordinates and isotropic or equivalent isotropic displacement parameters $\left(\AA^{2}\right)$

\begin{tabular}{|c|c|c|c|c|c|}
\hline & $x$ & $y$ & $z$ & $U_{\text {iso }} * / U_{\text {eq }}$ & Occ. $(<1)$ \\
\hline $\mathrm{O} 1$ & $0.72180(10)$ & $0.50889(10)$ & $0.48264(7)$ & $0.0392(3)$ & \\
\hline H1 & $0.7503(17)$ & $0.4189(12)$ & $0.5019(14)$ & $0.059^{*}$ & \\
\hline N1 & $0.67378(13)$ & $0.84618(14)$ & $0.18771(10)$ & $0.0460(3)$ & \\
\hline $\mathrm{H} 1 \mathrm{~A}$ & $0.6832(18)$ & $0.8918(18)$ & $0.1255(10)$ & $0.055^{*}$ & \\
\hline $\mathrm{C} 1$ & $0.70586(13)$ & $0.68167(13)$ & $0.33046(10)$ & $0.0311(3)$ & \\
\hline $\mathrm{C} 2$ & $0.77773(13)$ & $0.56216(13)$ & $0.39398(9)$ & $0.0313(3)$ & \\
\hline $\mathrm{C} 3$ & $0.90077(14)$ & $0.50210(15)$ & $0.36388(11)$ & $0.0387(3)$ & \\
\hline $\mathrm{H} 3$ & 0.949534 & 0.424117 & 0.406353 & $0.046^{*}$ & \\
\hline $\mathrm{C} 4$ & $0.95365(15)$ & $0.55642(17)$ & $0.27050(12)$ & $0.0451(3)$ & \\
\hline $\mathrm{H} 4$ & 1.036369 & 0.513198 & 0.252150 & $0.054^{*}$ & \\
\hline $\mathrm{C} 5$ & $0.88612(15)$ & $0.67154(16)$ & $0.20592(12)$ & $0.0441(3)$ & \\
\hline H5 & 0.920870 & 0.707054 & 0.143824 & $0.053^{*}$ & \\
\hline C6 & $0.76258(14)$ & $0.73365(14)$ & $0.23720(10)$ & $0.0364(3)$ & \\
\hline $\mathrm{C} 7$ & $0.56146(15)$ & $0.86403(16)$ & $0.24481(12)$ & $0.0440(3)$ & \\
\hline $\mathrm{H} 7$ & 0.486086 & 0.932698 & 0.225640 & $0.053^{*}$ & \\
\hline $\mathrm{C} 8$ & $0.57603(14)$ & $0.76694(14)$ & $0.33354(11)$ & $0.0356(3)$ & \\
\hline C9 & $0.4767(3)$ & $0.7551(3)$ & $0.4179(3)$ & $0.0401(6)$ & $0.895(3)$ \\
\hline H9A & 0.512632 & 0.820479 & 0.481067 & $0.048^{*}$ & $0.895(3)$ \\
\hline H9B & 0.478389 & 0.652079 & 0.445479 & $0.048^{*}$ & $0.895(3)$ \\
\hline $\mathrm{C} 10$ & $0.32164(18)$ & $0.7988(2)$ & $0.36920(16)$ & $0.0426(4)$ & $0.895(3)$ \\
\hline H10A & 0.318567 & 0.904813 & 0.348540 & $0.051^{*}$ & $0.895(3)$ \\
\hline H10B & 0.288874 & 0.740378 & 0.301680 & $0.051^{*}$ & $0.895(3)$ \\
\hline $\mathrm{N} 2$ & $0.22260(16)$ & $0.77279(16)$ & $0.44818(14)$ & $0.0414(4)$ & $0.895(3)$ \\
\hline $\mathrm{H} 2$ & $0.1338(10)$ & $0.778(2)$ & $0.4114(8)$ & $0.050^{*}$ & $0.895(3)$ \\
\hline $\mathrm{C} 11$ & $0.2400(3)$ & $0.8822(2)$ & $0.54022(17)$ & $0.0644(6)$ & $0.895(3)$ \\
\hline H11A & 0.233857 & 0.982920 & 0.510261 & $0.097 *$ & $0.895(3)$ \\
\hline H11B & 0.332532 & 0.867982 & 0.587682 & $0.097 *$ & $0.895(3)$ \\
\hline $\mathrm{H} 11 \mathrm{C}$ & 0.164866 & 0.867360 & 0.583032 & $0.097 *$ & $0.895(3)$ \\
\hline $\mathrm{C} 9^{\prime}$ & $0.477(3)$ & $0.724(3)$ & $0.410(3)$ & $0.0401(6)$ & $0.105(3)$ \\
\hline H9'A & 0.533638 & 0.706474 & 0.484119 & $0.048^{*}$ & $0.105(3)$ \\
\hline H9'B & 0.430684 & 0.628565 & 0.384330 & $0.048^{*}$ & $0.105(3)$ \\
\hline $\mathrm{C} 10^{\prime}$ & $0.3608(18)$ & $0.8382(19)$ & $0.4191(15)$ & $0.050(2)$ & $0.105(3)$ \\
\hline H10C & 0.405248 & 0.936642 & 0.435346 & $0.060^{*}$ & $0.105(3)$ \\
\hline H10D & 0.294887 & 0.844927 & 0.347824 & $0.060^{*}$ & $0.105(3)$ \\
\hline $\mathrm{N} 2^{\prime}$ & $0.2784(14)$ & $0.7990(17)$ & $0.5074(13)$ & $0.053(3)$ & $0.105(3)$ \\
\hline $\mathrm{H} 2^{\prime}$ & $0.256(4)$ & $0.703(2)$ & $0.500(4)$ & $0.063^{*}$ & $0.105(3)$ \\
\hline $\mathrm{C} 11^{\prime}$ & $0.143(2)$ & $0.884(3)$ & $0.507(2)$ & $0.083(6)$ & $0.105(3)$ \\
\hline H11D & 0.098734 & 0.849865 & 0.567605 & $0.125^{*}$ & $0.105(3)$ \\
\hline H11E & 0.165025 & 0.990058 & 0.516272 & $0.125^{*}$ & $0.105(3)$ \\
\hline
\end{tabular}




\begin{tabular}{llllll} 
H11F & 0.078492 & 0.868185 & 0.437255 & $0.125^{*}$ & $0.105(3)$ \\
\hline
\end{tabular}

Atomic displacement parameters $\left(\AA^{2}\right)$

\begin{tabular}{lllllll}
\hline & $U^{11}$ & $U^{22}$ & $U^{33}$ & $U^{12}$ & $U^{13}$ & $U^{23}$ \\
\hline $\mathrm{O} 1$ & $0.0534(6)$ & $0.0345(5)$ & $0.0337(5)$ & $0.0108(4)$ & $0.0185(4)$ & $0.0063(4)$ \\
$\mathrm{N} 1$ & $0.0523(7)$ & $0.0466(7)$ & $0.0415(6)$ & $0.0002(5)$ & $0.0151(5)$ & $0.0174(5)$ \\
$\mathrm{C} 1$ & $0.0332(6)$ & $0.0300(6)$ & $0.0311(6)$ & $-0.0049(5)$ & $0.0081(5)$ & $-0.0009(4)$ \\
$\mathrm{C} 2$ & $0.0354(6)$ & $0.0311(6)$ & $0.0279(6)$ & $-0.0023(5)$ & $0.0074(4)$ & $-0.0021(4)$ \\
$\mathrm{C} 3$ & $0.0362(7)$ & $0.0393(7)$ & $0.0407(7)$ & $0.0042(5)$ & $0.0074(5)$ & $0.0000(5)$ \\
$\mathrm{C} 4$ & $0.0368(7)$ & $0.0494(8)$ & $0.0536(8)$ & $-0.0024(6)$ & $0.0199(6)$ & $-0.0049(6)$ \\
$\mathrm{C} 5$ & $0.0448(7)$ & $0.0475(8)$ & $0.0451(7)$ & $-0.0096(6)$ & $0.0218(6)$ & $0.0017(6)$ \\
$\mathrm{C} 6$ & $0.0385(7)$ & $0.0359(6)$ & $0.0357(6)$ & $-0.0072(5)$ & $0.0097(5)$ & $0.0025(5)$ \\
$\mathrm{C} 7$ & $0.0448(7)$ & $0.0419(7)$ & $0.0463(7)$ & $0.0058(6)$ & $0.0109(6)$ & $0.0132(6)$ \\
$\mathrm{C} 8$ & $0.0372(6)$ & $0.0334(6)$ & $0.0374(6)$ & $0.0012(5)$ & $0.0097(5)$ & $0.0051(5)$ \\
$\mathrm{C} 9$ & $0.0448(7)$ & $0.0372(15)$ & $0.0414(10)$ & $0.0089(9)$ & $0.0160(6)$ & $0.0076(10)$ \\
C10 & $0.0412(9)$ & $0.0473(10)$ & $0.0411(9)$ & $0.0100(7)$ & $0.0124(7)$ & $0.0124(7)$ \\
N2 & $0.0383(7)$ & $0.0431(8)$ & $0.0450(8)$ & $0.0085(6)$ & $0.0132(6)$ & $0.0071(6)$ \\
C11 & $0.0799(15)$ & $0.0521(11)$ & $0.0699(13)$ & $0.0053(11)$ & $0.0368(11)$ & $-0.0077(10)$ \\
C9 ${ }^{\prime}$ & $0.0448(7)$ & $0.0372(15)$ & $0.0414(10)$ & $0.0089(9)$ & $0.0160(6)$ & $0.0076(10)$ \\
C10' & $0.053(5)$ & $0.046(4)$ & $0.053(5)$ & $0.007(4)$ & $0.015(4)$ & $0.005(4)$ \\
N2' & $0.059(5)$ & $0.046(5)$ & $0.059(6)$ & $0.007(5)$ & $0.026(5)$ & $0.001(5)$ \\
C11' & $0.066(10)$ & $0.078(11)$ & $0.112(13)$ & $0.024(9)$ & $0.038(9)$ & $0.027(10)$ \\
& & & & & & \\
\hline
\end{tabular}

Geometric parameters $\left(\AA,^{\circ}\right)$

\begin{tabular}{llll}
\hline $\mathrm{O} 1-\mathrm{H} 1$ & $0.858(9)$ & $\mathrm{C} 9-\mathrm{C} 10$ & $1.520(3)$ \\
$\mathrm{O} 1-\mathrm{C} 2$ & $1.3662(14)$ & $\mathrm{C} 10-\mathrm{H} 10 \mathrm{~A}$ & 0.9700 \\
$\mathrm{~N} 1-\mathrm{H} 1 \mathrm{~A}$ & $0.875(9)$ & $\mathrm{C} 10-\mathrm{H} 10 \mathrm{~B}$ & 0.9700 \\
$\mathrm{~N} 1-\mathrm{C} 6$ & $1.3655(18)$ & $\mathrm{C} 10-\mathrm{N} 2$ & $1.4729(19)$ \\
$\mathrm{N} 1-\mathrm{C} 7$ & $1.3753(18)$ & $\mathrm{N} 2-\mathrm{H} 2$ & $0.873(9)$ \\
$\mathrm{C} 1-\mathrm{C} 2$ & $1.4061(17)$ & $\mathrm{N} 2-\mathrm{C} 11$ & $1.464(2)$ \\
$\mathrm{C} 1-\mathrm{C} 6$ & $1.4147(17)$ & $\mathrm{C} 11-\mathrm{H} 11 \mathrm{~A}$ & 0.9600 \\
$\mathrm{C} 1-\mathrm{C} 8$ & $1.4416(17)$ & $\mathrm{C} 11-\mathrm{H} 11 \mathrm{~B}$ & 0.9600 \\
$\mathrm{C} 2-\mathrm{C} 3$ & $1.3823(18)$ & $\mathrm{C} 11-\mathrm{H} 11 \mathrm{C}$ & 0.9600 \\
$\mathrm{C} 3-\mathrm{H} 3$ & 0.9300 & $\mathrm{C} 9^{\prime}-\mathrm{H} 9^{\prime} \mathrm{A}$ & 0.9700 \\
$\mathrm{C} 3-\mathrm{C} 4$ & $1.4042(19)$ & $\mathrm{C} 9^{\prime}-\mathrm{H} 9^{\prime} \mathrm{B}$ & 0.9700 \\
$\mathrm{C} 4-\mathrm{H} 4$ & 0.9300 & $\mathrm{C} 9^{\prime}-\mathrm{C} 10^{\prime}$ & $1.509(10)$ \\
$\mathrm{C} 4-\mathrm{C} 5$ & $1.368(2)$ & $\mathrm{C} 10^{\prime}-\mathrm{H} 10 \mathrm{C}$ & 0.9700 \\
$\mathrm{C} 5-\mathrm{H} 5$ & 0.9300 & $\mathrm{C} 10^{\prime}-\mathrm{H} 10 \mathrm{D}$ & 0.9700 \\
$\mathrm{C} 5-\mathrm{C} 6$ & $1.3995(19)$ & $\mathrm{C} 10^{\prime}-\mathrm{N} 2^{\prime}$ & $1.476(9)$ \\
$\mathrm{C} 7-\mathrm{H} 7$ & 0.9300 & $\mathrm{~N} 2^{\prime}-\mathrm{H} 2^{\prime}$ & $0.876(14)$ \\
$\mathrm{C} 7-\mathrm{C} 8$ & $1.3649(18)$ & $\mathrm{N} 2^{\prime}-\mathrm{C} 11^{\prime}$ & $1.476(10)$ \\
$\mathrm{C} 8-\mathrm{C} 9$ & $1.512(3)$ & $\mathrm{C} 11^{\prime}-\mathrm{H} 11 \mathrm{D}$ & 0.9600 \\
$\mathrm{C} 8-\mathrm{C} 9^{\prime}$ & $1.48(3)$ & $\mathrm{C} 11^{\prime}-\mathrm{H} 11 \mathrm{E}$ & 0.9600 \\
$\mathrm{C} 9-\mathrm{H} 9 \mathrm{~A}$ & 0.9700 & $\mathrm{C} 11^{\prime}-\mathrm{H} 11 \mathrm{~F}$ & 0.9600 \\
$\mathrm{C} 9-\mathrm{H} 9 \mathrm{~B}$ & 0.9700 & &
\end{tabular}




\begin{tabular}{|c|c|c|c|}
\hline $\mathrm{C} 2-\mathrm{O} 1-\mathrm{H} 1$ & $112.8(11)$ & $\mathrm{C} 9-\mathrm{C} 10-\mathrm{H} 10 \mathrm{~A}$ & 109.1 \\
\hline $\mathrm{C} 6-\mathrm{N} 1-\mathrm{H} 1 \mathrm{~A}$ & $124.4(12)$ & $\mathrm{C} 9-\mathrm{C} 10-\mathrm{H} 10 \mathrm{~B}$ & 109.1 \\
\hline $\mathrm{C} 6-\mathrm{N} 1-\mathrm{C} 7$ & $109.04(11)$ & $\mathrm{H} 10 \mathrm{~A}-\mathrm{C} 10-\mathrm{H} 10 \mathrm{~B}$ & 107.8 \\
\hline $\mathrm{C} 7-\mathrm{N} 1-\mathrm{H} 1 \mathrm{~A}$ & $126.3(11)$ & $\mathrm{N} 2-\mathrm{C} 10-\mathrm{C} 9$ & $112.57(17)$ \\
\hline $\mathrm{C} 2-\mathrm{C} 1-\mathrm{C} 6$ & $118.00(11)$ & $\mathrm{N} 2-\mathrm{C} 10-\mathrm{H} 10 \mathrm{~A}$ & 109.1 \\
\hline $\mathrm{C} 2-\mathrm{C} 1-\mathrm{C} 8$ & $134.67(11)$ & $\mathrm{N} 2-\mathrm{C} 10-\mathrm{H} 10 \mathrm{~B}$ & 109.1 \\
\hline $\mathrm{C} 6-\mathrm{C} 1-\mathrm{C} 8$ & $107.27(11)$ & $\mathrm{C} 10-\mathrm{N} 2-\mathrm{H} 2$ & $108.6(7)$ \\
\hline $\mathrm{O} 1-\mathrm{C} 2-\mathrm{C} 1$ & $118.40(10)$ & $\mathrm{C} 11-\mathrm{N} 2-\mathrm{C} 10$ & $113.51(15)$ \\
\hline $\mathrm{O} 1-\mathrm{C} 2-\mathrm{C} 3$ & $122.57(11)$ & $\mathrm{C} 11-\mathrm{N} 2-\mathrm{H} 2$ & $108.6(7)$ \\
\hline $\mathrm{C} 3-\mathrm{C} 2-\mathrm{C} 1$ & $119.03(11)$ & $\mathrm{N} 2-\mathrm{C} 11-\mathrm{H} 11 \mathrm{~A}$ & 109.5 \\
\hline $\mathrm{C} 2-\mathrm{C} 3-\mathrm{H} 3$ & 119.3 & $\mathrm{~N} 2-\mathrm{C} 11-\mathrm{H} 11 \mathrm{~B}$ & 109.5 \\
\hline $\mathrm{C} 2-\mathrm{C} 3-\mathrm{C} 4$ & $121.35(13)$ & $\mathrm{N} 2-\mathrm{C} 11-\mathrm{H} 11 \mathrm{C}$ & 109.5 \\
\hline $\mathrm{C} 4-\mathrm{C} 3-\mathrm{H} 3$ & 119.3 & $\mathrm{H} 11 \mathrm{~A}-\mathrm{C} 11-\mathrm{H} 11 \mathrm{~B}$ & 109.5 \\
\hline $\mathrm{C} 3-\mathrm{C} 4-\mathrm{H} 4$ & 119.3 & $\mathrm{H} 11 \mathrm{~A}-\mathrm{C} 11-\mathrm{H} 11 \mathrm{C}$ & 109.5 \\
\hline $\mathrm{C} 5-\mathrm{C} 4-\mathrm{C} 3$ & $121.43(13)$ & $\mathrm{H} 11 \mathrm{~B}-\mathrm{C} 11-\mathrm{H} 11 \mathrm{C}$ & 109.5 \\
\hline $\mathrm{C} 5-\mathrm{C} 4-\mathrm{H} 4$ & 119.3 & $\mathrm{C} 8-\mathrm{C}^{\prime}-\mathrm{H} 9^{\prime} \mathrm{A}$ & 108.6 \\
\hline $\mathrm{C} 4-\mathrm{C} 5-\mathrm{H} 5$ & 121.4 & $\mathrm{C} 8-\mathrm{C}^{\prime}-\mathrm{H} 9^{\prime} \mathrm{B}$ & 108.6 \\
\hline $\mathrm{C} 4-\mathrm{C} 5-\mathrm{C} 6$ & $117.23(12)$ & $\mathrm{C} 8-\mathrm{C}^{\prime}-\mathrm{C} 10^{\prime}$ & $115(2)$ \\
\hline $\mathrm{C} 6-\mathrm{C} 5-\mathrm{H} 5$ & 121.4 & $\mathrm{H} 9^{\prime} \mathrm{A}-\mathrm{C} 9^{\prime}-\mathrm{H} 9^{\prime} \mathrm{B}$ & 107.6 \\
\hline $\mathrm{N} 1-\mathrm{C} 6-\mathrm{C} 1$ & $107.40(11)$ & $\mathrm{C} 10^{\prime}-\mathrm{C} 9^{\prime}-\mathrm{H} 9^{\prime} \mathrm{A}$ & 108.6 \\
\hline $\mathrm{N} 1-\mathrm{C} 6-\mathrm{C} 5$ & $129.63(12)$ & $\mathrm{C} 10^{\prime}-\mathrm{C} 9^{\prime}-\mathrm{H} 9^{\prime} \mathrm{B}$ & 108.6 \\
\hline $\mathrm{C} 5-\mathrm{C} 6-\mathrm{C} 1$ & $122.96(12)$ & $\mathrm{C} 9^{\prime}-\mathrm{C} 10^{\prime}-\mathrm{H} 10 \mathrm{C}$ & 109.1 \\
\hline $\mathrm{N} 1-\mathrm{C} 7-\mathrm{H} 7$ & 124.7 & $\mathrm{C} 9^{\prime}-\mathrm{C} 10^{\prime}-\mathrm{H} 10 \mathrm{D}$ & 109.1 \\
\hline $\mathrm{C} 8-\mathrm{C} 7-\mathrm{N} 1$ & $110.55(12)$ & $\mathrm{H} 10 \mathrm{C}-\mathrm{C} 10^{\prime}-\mathrm{H} 10 \mathrm{D}$ & 107.9 \\
\hline $\mathrm{C} 8-\mathrm{C} 7-\mathrm{H} 7$ & 124.7 & $\mathrm{~N} 2^{\prime}-\mathrm{C} 10^{\prime}-\mathrm{C} 9^{\prime}$ & $112.3(16)$ \\
\hline $\mathrm{C} 1-\mathrm{C} 8-\mathrm{C} 9$ & $127.83(14)$ & $\mathrm{N} 2^{\prime}-\mathrm{C} 10^{\prime}-\mathrm{H} 10 \mathrm{C}$ & 109.1 \\
\hline $\mathrm{C} 1-\mathrm{C} 8-\mathrm{C} 9^{\prime}$ & $120.9(8)$ & $\mathrm{N} 2^{\prime}-\mathrm{C} 10^{\prime}-\mathrm{H} 10 \mathrm{D}$ & 109.1 \\
\hline $\mathrm{C} 7-\mathrm{C} 8-\mathrm{C} 1$ & $105.73(11)$ & $\mathrm{C} 10^{\prime}-\mathrm{N} 2^{\prime}-\mathrm{H} 2^{\prime}$ & $107.7(14)$ \\
\hline $\mathrm{C} 7-\mathrm{C} 8-\mathrm{C} 9$ & $126.43(15)$ & $\mathrm{C} 10^{\prime}-\mathrm{N} 2^{\prime}-\mathrm{C} 11^{\prime}$ & $116.4(13)$ \\
\hline $\mathrm{C} 7-\mathrm{C} 8-\mathrm{C} 9^{\prime}$ & $132.4(9)$ & $\mathrm{C} 11^{\prime}-\mathrm{N} 2^{\prime}-\mathrm{H} 2^{\prime}$ & $107.6(14)$ \\
\hline $\mathrm{C} 8-\mathrm{C} 9-\mathrm{H} 9 \mathrm{~A}$ & 109.0 & $\mathrm{~N} 2{ }^{\prime}-\mathrm{C} 11^{\prime}-\mathrm{H} 11 \mathrm{D}$ & 109.5 \\
\hline $\mathrm{C} 8-\mathrm{C} 9-\mathrm{H} 9 \mathrm{~B}$ & 109.0 & $\mathrm{~N} 2{ }^{\prime}-\mathrm{C} 11^{\prime}-\mathrm{H} 11 \mathrm{E}$ & 109.5 \\
\hline $\mathrm{C} 8-\mathrm{C} 9-\mathrm{C} 10$ & $112.8(2)$ & $\mathrm{N} 2{ }^{\prime}-\mathrm{C} 11^{\prime}-\mathrm{H} 11 \mathrm{~F}$ & 109.5 \\
\hline $\mathrm{H} 9 \mathrm{~A}-\mathrm{C} 9-\mathrm{H} 9 \mathrm{~B}$ & 107.8 & $\mathrm{H} 11 \mathrm{D}-\mathrm{C} 11^{\prime}-\mathrm{H} 11 \mathrm{E}$ & 109.5 \\
\hline $\mathrm{C} 10-\mathrm{C} 9-\mathrm{H} 9 \mathrm{~A}$ & 109.0 & $\mathrm{H} 11 \mathrm{D}-\mathrm{C} 11^{\prime}-\mathrm{H} 11 \mathrm{~F}$ & 109.5 \\
\hline $\mathrm{C} 10-\mathrm{C} 9-\mathrm{H} 9 \mathrm{~B}$ & 109.0 & $\mathrm{H} 11 \mathrm{E}-\mathrm{C} 11^{\prime}-\mathrm{H} 11 \mathrm{~F}$ & 109.5 \\
\hline $\mathrm{O} 1-\mathrm{C} 2-\mathrm{C} 3-\mathrm{C} 4$ & $178.26(11)$ & $\mathrm{C} 6-\mathrm{C} 1-\mathrm{C} 2-\mathrm{O} 1$ & $-178.50(10)$ \\
\hline $\mathrm{N} 1-\mathrm{C} 7-\mathrm{C} 8-\mathrm{C} 1$ & $-0.66(16)$ & $\mathrm{C} 6-\mathrm{C} 1-\mathrm{C} 2-\mathrm{C} 3$ & $0.80(17)$ \\
\hline $\mathrm{N} 1-\mathrm{C} 7-\mathrm{C} 8-\mathrm{C} 9$ & $178.33(19)$ & $\mathrm{C} 6-\mathrm{C} 1-\mathrm{C} 8-\mathrm{C} 7$ & $-0.28(14)$ \\
\hline $\mathrm{N} 1-\mathrm{C} 7-\mathrm{C} 8-\mathrm{C} 9^{\prime}$ & $-169(2)$ & $\mathrm{C} 6-\mathrm{C} 1-\mathrm{C} 8-\mathrm{C} 9$ & $-179.24(18)$ \\
\hline $\mathrm{C} 1-\mathrm{C} 2-\mathrm{C} 3-\mathrm{C} 4$ & $-1.01(19)$ & $\mathrm{C} 6-\mathrm{C} 1-\mathrm{C} 8-\mathrm{C} 9^{\prime}$ & $169.9(18)$ \\
\hline $\mathrm{C} 1-\mathrm{C} 8-\mathrm{C} 9-\mathrm{C} 10$ & $-151.89(16)$ & $\mathrm{C} 7-\mathrm{N} 1-\mathrm{C} 6-\mathrm{C} 1$ & $-1.52(15)$ \\
\hline $\mathrm{C} 1-\mathrm{C} 8-\mathrm{C} 9^{\prime}-\mathrm{C} 10^{\prime}$ & $169.5(18)$ & $\mathrm{C} 7-\mathrm{N} 1-\mathrm{C} 6-\mathrm{C} 5$ & $176.94(14)$ \\
\hline $\mathrm{C} 2-\mathrm{C} 1-\mathrm{C} 6-\mathrm{N} 1$ & $178.58(11)$ & $\mathrm{C} 7-\mathrm{C} 8-\mathrm{C} 9-\mathrm{C} 10$ & $29.3(3)$ \\
\hline $\mathrm{C} 2-\mathrm{C} 1-\mathrm{C} 6-\mathrm{C} 5$ & $-0.01(19)$ & $\mathrm{C} 7-\mathrm{C} 8-\mathrm{C} 9^{\prime}-\mathrm{C} 10^{\prime}$ & $-23(4)$ \\
\hline $\mathrm{C} 2-\mathrm{C} 1-\mathrm{C} 8-\mathrm{C} 7$ & $-177.14(14)$ & $\mathrm{C} 8-\mathrm{C} 1-\mathrm{C} 2-\mathrm{O} 1$ & $-1.9(2)$ \\
\hline $\mathrm{C} 2-\mathrm{C} 1-\mathrm{C} 8-\mathrm{C} 9$ & $3.9(3)$ & $\mathrm{C} 8-\mathrm{C} 1-\mathrm{C} 2-\mathrm{C} 3$ & $177.41(13)$ \\
\hline
\end{tabular}




$\begin{array}{llll}\mathrm{C} 2-\mathrm{C} 1-\mathrm{C} 8-\mathrm{C} 9^{\prime} & -7.0(18) & \mathrm{C} 8-\mathrm{C} 1-\mathrm{C} 6-\mathrm{N} 1 & 1.11(14) \\ \mathrm{C} 2-\mathrm{C} 3-\mathrm{C} 4-\mathrm{C} 5 & 0.4(2) & \mathrm{C} 8-\mathrm{C} 1-\mathrm{C} 6-\mathrm{C} 5 & -177.48(12) \\ \mathrm{C} 3-\mathrm{C} 4-\mathrm{C} 5-\mathrm{C} 6 & 0.4(2) & \mathrm{C} 8-\mathrm{C} 9-\mathrm{C} 10-\mathrm{N} 2 & 174.34(15) \\ \mathrm{C} 4-\mathrm{C} 5-\mathrm{C} 6-\mathrm{N} 1 & -178.84(14) & \mathrm{C} 8-\mathrm{C} 9^{\prime}-\mathrm{C} 10^{\prime}-\mathrm{N} 2^{\prime} & -172(2) \\ \mathrm{C} 4-\mathrm{C} 5-\mathrm{C} 6-\mathrm{C} 1 & -0.6(2) & \mathrm{C} 9-\mathrm{C} 10-\mathrm{N} 2-\mathrm{C} 11 & 73.1(2) \\ \mathrm{C} 6-\mathrm{N} 1-\mathrm{C} 7-\mathrm{C} 8 & 1.39(17) & \mathrm{C} 9^{\prime}-\mathrm{C} 10^{\prime}-\mathrm{N} 2^{\prime}-\mathrm{C} 11^{\prime} & -167(3)\end{array}$

Hydrogen-bond geometry $\left(\AA,{ }^{\circ}\right)$

\begin{tabular}{lllll}
\hline$D-\mathrm{H} \cdots A$ & $D-\mathrm{H}$ & $\mathrm{H} \cdots A$ & $D \cdots A$ & $D-\mathrm{H} \cdots A$ \\
\hline $\mathrm{O} 1-\mathrm{H} 1 \cdots \mathrm{N} 2^{\mathrm{i}}$ & $0.86(1)$ & $1.80(1)$ & $2.6501(16)$ & $169(2)$ \\
$\mathrm{N} 1-\mathrm{H} 1 A \cdots \mathrm{O} 1^{\mathrm{ii}}$ & $0.88(1)$ & $2.04(1)$ & $2.9092(15)$ & $175(2)$ \\
\hline
\end{tabular}

Symmetry codes: (i) $-x+1,-y+1,-z+1$; (ii) $x,-y+3 / 2, z-1 / 2$.

Bis $\{[2-(4-h y d r o x y-1 H$-indol-3-yl)ethyl]methylazanium\} but-2-enedioate (II)

Crystal data

$\mathrm{C}_{11} \mathrm{H}_{15} \mathrm{~N}_{2} \mathrm{O}^{+} \cdot 0.5 \mathrm{C}_{4} \mathrm{H}_{2} \mathrm{O}_{4}{ }^{2-}$

$M_{r}=248.28$

Triclinic, $P \overline{1}$

$a=7.7363(10) \AA$

$b=9.7146(12) \AA$

$c=9.7854(13) \AA$

$\alpha=105.524(4)^{\circ}$

$\beta=110.554(4)^{\circ}$

$\gamma=97.167(4)^{\circ}$

$V=643.69(14) \AA^{3}$

\section{Data collection}

Bruker D8 Venture CMOS diffractometer

$\varphi$ and $\omega$ scans

Absorption correction: multi-scan

(SADABS; Bruker, 2018)

$T_{\min }=0.685, T_{\max }=0.745$

14395 measured reflections

\section{Refinement}

Refinement on $F^{2}$

Least-squares matrix: full

$R\left[F^{2}>2 \sigma\left(F^{2}\right)\right]=0.039$

$w R\left(F^{2}\right)=0.098$

$S=1.11$

2365 reflections

181 parameters

4 restraints

Hydrogen site location: mixed
$Z=2$

$F(000)=264$

$D_{\mathrm{x}}=1.281 \mathrm{Mg} \mathrm{m}^{-3}$

Mo $K \alpha$ radiation, $\lambda=0.71073 \AA$

Cell parameters from 3848 reflections

$\theta=2.7-25.5^{\circ}$

$\mu=0.09 \mathrm{~mm}^{-1}$

$T=297 \mathrm{~K}$

BLOCK, colourless

$0.24 \times 0.19 \times 0.03 \mathrm{~mm}$

2365 independent reflections

1774 reflections with $I>2 \sigma(I)$

$R_{\text {int }}=0.046$

$\theta_{\text {max }}=25.5^{\circ}, \theta_{\text {min }}=2.7^{\circ}$

$h=-9 \rightarrow 9$

$k=-11 \rightarrow 11$

$l=-11 \rightarrow 11$

$\mathrm{H}$ atoms treated by a mixture of independent and constrained refinement

$w=1 /\left[\sigma^{2}\left(F_{\mathrm{o}}^{2}\right)+(0.0428 P)^{2}+0.1031 P\right]$

where $P=\left(F_{\mathrm{o}}^{2}+2 F_{\mathrm{c}}^{2}\right) / 3$

$(\Delta / \sigma)_{\max }<0.001$

$\Delta \rho_{\max }=0.15$ e $\AA^{-3}$

$\Delta \rho_{\text {min }}=-0.15$ e $\AA^{-3}$

Extinction correction: SHELXL2018

(Sheldrick, 2015b),

$\mathrm{Fc}^{*}=\mathrm{kFc}\left[1+0.001 \times \mathrm{xc}^{2} \lambda^{3} / \sin (2 \theta)\right]^{-1 / 4}$

Extinction coefficient: 0.035 (8) 


\section{Special details}

Geometry. All esds (except the esd in the dihedral angle between two 1.s. planes) are estimated using the full covariance matrix. The cell esds are taken into account individually in the estimation of esds in distances, angles and torsion angles; correlations between esds in cell parameters are only used when they are defined by crystal symmetry. An approximate (isotropic) treatment of cell esds is used for estimating esds involving l.s. planes.

Fractional atomic coordinates and isotropic or equivalent isotropic displacement parameters $\left(\AA^{2}\right)$

\begin{tabular}{|c|c|c|c|c|}
\hline & $x$ & $y$ & $z$ & $U_{\text {iso }} * / U_{\text {eq }}$ \\
\hline O1 & $0.71021(18)$ & $0.24171(13)$ & $0.73074(16)$ & $0.0516(4)$ \\
\hline N1 & $1.1025(2)$ & $0.68290(16)$ & $0.82025(18)$ & $0.0458(4)$ \\
\hline $\mathrm{N} 2$ & $1.1755(2)$ & $0.13836(16)$ & $0.45820(17)$ & $0.0390(4)$ \\
\hline $\mathrm{C} 1$ & $0.9034(2)$ & $0.47406(17)$ & $0.77976(18)$ & $0.0351(4)$ \\
\hline $\mathrm{C} 2$ & $0.7537(2)$ & $0.39203(18)$ & $0.79685(19)$ & $0.0391(4)$ \\
\hline C3 & $0.6597(3)$ & $0.4650(2)$ & $0.8775(2)$ & $0.0492(5)$ \\
\hline H3 & 0.559823 & 0.411333 & 0.888131 & $0.059^{*}$ \\
\hline $\mathrm{C} 4$ & $0.7117(3)$ & $0.6188(2)$ & $0.9441(2)$ & $0.0532(5)$ \\
\hline H4 & 0.644580 & 0.664964 & 0.997328 & $0.064^{*}$ \\
\hline $\mathrm{C} 5$ & $0.8583(3)$ & $0.7030(2)$ & $0.9328(2)$ & $0.0480(5)$ \\
\hline H5 & 0.893452 & 0.804937 & 0.978251 & $0.058^{*}$ \\
\hline C6 & $0.9524(2)$ & $0.62851(18)$ & $0.85004(19)$ & $0.0391(4)$ \\
\hline $\mathrm{C} 7$ & $1.1455(3)$ & 0.56779 (19) & $0.7313(2)$ & $0.0444(4)$ \\
\hline H7 & 1.240746 & 0.577663 & 0.694858 & $0.053^{*}$ \\
\hline $\mathrm{C} 8$ & $1.0291(2)$ & $0.43736(18)$ & $0.70422(19)$ & $0.0379(4)$ \\
\hline C9 & $1.0327(3)$ & $0.28658(18)$ & $0.6159(2)$ & $0.0434(4)$ \\
\hline H9A & 0.906459 & 0.236367 & 0.536418 & $0.052^{*}$ \\
\hline H9B & 1.066169 & 0.230581 & 0.685761 & $0.052^{*}$ \\
\hline $\mathrm{C} 10$ & $1.1729(2)$ & $0.29082(18)$ & $0.5407(2)$ & $0.0398(4)$ \\
\hline $\mathrm{H} 10 \mathrm{~A}$ & 1.137504 & 0.343594 & 0.467796 & $0.048^{*}$ \\
\hline H10B & 1.299028 & 0.342809 & 0.619295 & $0.048^{*}$ \\
\hline C11 & $1.3055(3)$ & $0.1317(2)$ & $0.3774(2)$ & $0.0561(5)$ \\
\hline H11A & 1.293563 & 0.030805 & 0.321818 & $0.084^{*}$ \\
\hline H11B & 1.434183 & 0.174879 & 0.451983 & $0.084 *$ \\
\hline $\mathrm{H} 11 \mathrm{C}$ & 1.273049 & 0.184976 & 0.306086 & $0.084^{*}$ \\
\hline $\mathrm{C} 12$ & $0.3434(2)$ & $0.02114(16)$ & $0.80503(17)$ & $0.0311(4)$ \\
\hline $\mathrm{C} 13$ & $0.5073(2)$ & $0.01566(17)$ & $0.94080(18)$ & $0.0344(4)$ \\
\hline H13 & 0.628166 & 0.035974 & 0.941097 & $0.041^{*}$ \\
\hline $\mathrm{O} 2$ & $0.17836(15)$ & $-0.03196(13)$ & $0.78734(13)$ & $0.0423(3)$ \\
\hline $\mathrm{O} 3$ & $0.38449(16)$ & $0.07932(13)$ & $0.71675(14)$ & $0.0461(3)$ \\
\hline H1 & $0.613(2)$ & $0.203(2)$ & $0.745(3)$ & $0.074(7)^{*}$ \\
\hline $\mathrm{H} 1 \mathrm{~A}$ & $1.141(3)$ & $0.7760(12)$ & $0.839(2)$ & $0.069(7)^{*}$ \\
\hline $\mathrm{H} 2 \mathrm{~A}$ & $1.216(3)$ & $0.0955(19)$ & $0.5298(18)$ & $0.054(6)^{*}$ \\
\hline $\mathrm{H} 2 \mathrm{~B}$ & $1.0586(16)$ & $0.0926(19)$ & $0.3889(18)$ & $0.054(6) *$ \\
\hline
\end{tabular}

Atomic displacement parameters $\left(\AA^{2}\right)$

\begin{tabular}{lllllll}
\hline & $U^{11}$ & $U^{22}$ & $U^{33}$ & $U^{12}$ & $U^{13}$ & $U^{23}$ \\
\hline $\mathrm{O} 1$ & $0.0481(8)$ & $0.0429(7)$ & $0.0624(9)$ & $-0.0009(6)$ & $0.0278(7)$ & $0.0135(6)$
\end{tabular}




\begin{tabular}{lllllll}
$\mathrm{N} 1$ & $0.0510(9)$ & $0.0347(8)$ & $0.0500(9)$ & $0.0033(7)$ & $0.0194(8)$ & $0.0161(7)$ \\
$\mathrm{N} 2$ & $0.0369(8)$ & $0.0476(9)$ & $0.0337(8)$ & $0.0135(7)$ & $0.0111(7)$ & $0.0178(7)$ \\
$\mathrm{C} 1$ & $0.0342(9)$ & $0.0385(9)$ & $0.0306(9)$ & $0.0070(7)$ & $0.0094(7)$ & $0.0145(7)$ \\
$\mathrm{C} 2$ & $0.0376(9)$ & $0.0407(9)$ & $0.0353(9)$ & $0.0049(7)$ & $0.0115(8)$ & $0.0133(7)$ \\
$\mathrm{C} 3$ & $0.0429(10)$ & $0.0626(12)$ & $0.0477(11)$ & $0.0107(9)$ & $0.0233(9)$ & $0.0212(9)$ \\
$\mathrm{C} 4$ & $0.0609(12)$ & $0.0589(12)$ & $0.0486(12)$ & $0.0230(10)$ & $0.0304(10)$ & $0.0167(9)$ \\
$\mathrm{C} 5$ & $0.0625(12)$ & $0.0408(10)$ & $0.0409(10)$ & $0.0169(9)$ & $0.0199(9)$ & $0.0130(8)$ \\
C6 & $0.0419(9)$ & $0.0393(9)$ & $0.0348(9)$ & $0.0079(7)$ & $0.0114(8)$ & $0.0166(7)$ \\
C7 & $0.0433(10)$ & $0.0475(10)$ & $0.0468(11)$ & $0.0067(8)$ & $0.0217(9)$ & $0.0199(8)$ \\
C8 & $0.0369(9)$ & $0.0417(9)$ & $0.0363(9)$ & $0.0085(7)$ & $0.0139(8)$ & $0.0162(7)$ \\
C9 & $0.0430(10)$ & $0.0425(10)$ & $0.0457(10)$ & $0.0084(8)$ & $0.0201(9)$ & $0.0144(8)$ \\
C10 & $0.0387(9)$ & $0.0421(9)$ & $0.0389(10)$ & $0.0102(7)$ & $0.0132(8)$ & $0.0170(7)$ \\
C11 & $0.0576(12)$ & $0.0740(14)$ & $0.0527(12)$ & $0.0281(10)$ & $0.0307(10)$ & $0.0291(10)$ \\
C12 & $0.0330(9)$ & $0.0272(8)$ & $0.0294(8)$ & $0.0041(6)$ & $0.0086(7)$ & $0.0106(6)$ \\
C13 & $0.0287(8)$ & $0.0385(9)$ & $0.0343(9)$ & $0.0043(7)$ & $0.0097(7)$ & $0.0154(7)$ \\
O2 & $0.0297(6)$ & $0.0494(7)$ & $0.0429(7)$ & $0.0014(5)$ & $0.0060(5)$ & $0.0233(6)$ \\
O3 & $0.0399(7)$ & $0.0606(8)$ & $0.0412(7)$ & $0.0057(6)$ & $0.0114(6)$ & $0.0322(6)$ \\
& & & & & & \\
\hline
\end{tabular}

Geometric parameters $\left(A,{ }^{\circ}\right)$

\begin{tabular}{llll}
\hline $\mathrm{O} 1-\mathrm{C} 2$ & $1.372(2)$ & $\mathrm{C} 5-\mathrm{C} 6$ & $1.393(3)$ \\
$\mathrm{O} 1-\mathrm{H} 1$ & $0.870(10)$ & $\mathrm{C} 7-\mathrm{H} 7$ & 0.9300 \\
$\mathrm{~N} 1-\mathrm{C} 6$ & $1.372(2)$ & $\mathrm{C} 7-\mathrm{C} 8$ & $1.362(2)$ \\
$\mathrm{N} 1-\mathrm{C} 7$ & $1.376(2)$ & $\mathrm{C} 8-\mathrm{C} 9$ & $1.498(2)$ \\
$\mathrm{N} 1-\mathrm{H} 1 \mathrm{~A}$ & $0.863(10)$ & $\mathrm{C} 9-\mathrm{H} 9 \mathrm{~A}$ & 0.9700 \\
$\mathrm{~N} 2-\mathrm{C} 10$ & $1.492(2)$ & $\mathrm{C} 9-\mathrm{H} 9 \mathrm{~B}$ & 0.9700 \\
$\mathrm{~N} 2-\mathrm{C} 11$ & $1.479(2)$ & $\mathrm{C} 9-\mathrm{C} 10$ & $1.511(2)$ \\
$\mathrm{N} 2-\mathrm{H} 2 \mathrm{~A}$ & $0.892(9)$ & $\mathrm{C} 10-\mathrm{H} 10 \mathrm{~A}$ & 0.9700 \\
$\mathrm{~N} 2-\mathrm{H} 2 \mathrm{~B}$ & $0.885(9)$ & $\mathrm{C} 10-\mathrm{H} 10 \mathrm{~B}$ & 0.9700 \\
$\mathrm{C} 1-\mathrm{C} 2$ & $1.408(2)$ & $\mathrm{C} 11-\mathrm{H} 11 \mathrm{~A}$ & 0.9600 \\
$\mathrm{C} 1-\mathrm{C} 6$ & $1.411(2)$ & $\mathrm{C} 11-\mathrm{H} 11 \mathrm{~B}$ & 0.9600 \\
$\mathrm{C} 1-\mathrm{C} 8$ & $1.438(2)$ & $\mathrm{C} 11-\mathrm{H} 11 \mathrm{C}$ & 0.9600 \\
$\mathrm{C} 2-\mathrm{C} 3$ & $1.373(3)$ & $\mathrm{C} 12-\mathrm{C} 13$ & $1.499(2)$ \\
$\mathrm{C} 3-\mathrm{H} 3$ & 0.9300 & $\mathrm{C} 12-\mathrm{O} 2$ & $1.2488(18)$ \\
$\mathrm{C} 3-\mathrm{C} 4$ & $1.402(3)$ & $\mathrm{C} 12-\mathrm{O} 3$ & $1.2553(18)$ \\
$\mathrm{C} 4-\mathrm{H} 4$ & 0.9300 & $\mathrm{C} 13-\mathrm{C} 13$ & 0.9300 \\
$\mathrm{C} 4-\mathrm{C} 5$ & $1.368(3)$ & $\mathrm{C} 13-\mathrm{H} 13$ & \\
$\mathrm{C} 5-\mathrm{H} 5$ & 0.9300 & & $110.51(15)$ \\
& & $\mathrm{C} 8-\mathrm{C} 7-\mathrm{N} 1$ & 124.7 \\
$\mathrm{C} 2-\mathrm{O} 1-\mathrm{H} 1$ & $109.6(15)$ & $\mathrm{C} 8-\mathrm{C} 7-\mathrm{H} 7$ & $127.04(14)$ \\
$\mathrm{C} 6-\mathrm{N} 1-\mathrm{C} 7$ & $108.98(14)$ & $\mathrm{C} 1-\mathrm{C} 8-\mathrm{C} 9$ & $105.81(15)$ \\
$\mathrm{C} 6-\mathrm{N} 1-\mathrm{H} 1 \mathrm{~A}$ & $121.5(15)$ & $\mathrm{C} 7-\mathrm{C} 8-\mathrm{C} 1$ & $127.14(16)$ \\
$\mathrm{C} 7-\mathrm{N} 1-\mathrm{H} 1 \mathrm{~A}$ & $128.2(15)$ & $\mathrm{C} 8-\mathrm{C} 9-\mathrm{C} 9$ & 109.1 \\
$\mathrm{C} 10-\mathrm{N} 2-\mathrm{H} 2 \mathrm{~A}$ & $106.9(13)$ & $\mathrm{C} 8-\mathrm{C} 9-\mathrm{C} 10$ & 109.1 \\
$\mathrm{C} 10-\mathrm{N} 2-\mathrm{H} 2 \mathrm{~B}$ & $107.9(13)$ & $\mathrm{H} 9 \mathrm{~A}-\mathrm{C} 9-\mathrm{H} 9 \mathrm{~B}$ & $112.38(13)$ \\
$\mathrm{C} 11-\mathrm{N} 2-\mathrm{C} 10$ & $114.20(14)$ & & 107.9 \\
$\mathrm{C} 11-\mathrm{N} 2-\mathrm{H} 2 \mathrm{~A}$ & $107.5(13)$ & & \\
$\mathrm{C} 11-\mathrm{N} 2-\mathrm{H} 2 \mathrm{~B}$ & & &
\end{tabular}




\begin{tabular}{|c|c|c|c|}
\hline $\mathrm{H} 2 \mathrm{~A}-\mathrm{N} 2-\mathrm{H} 2 \mathrm{~B}$ & $112.1(18)$ & $\mathrm{C} 10-\mathrm{C} 9-\mathrm{H} 9 \mathrm{~A}$ & 109.1 \\
\hline $\mathrm{C} 2-\mathrm{C} 1-\mathrm{C} 6$ & $117.87(15)$ & $\mathrm{C} 10-\mathrm{C} 9-\mathrm{H} 9 \mathrm{~B}$ & 109.1 \\
\hline $\mathrm{C} 2-\mathrm{C} 1-\mathrm{C} 8$ & $134.53(15)$ & $\mathrm{N} 2-\mathrm{C} 10-\mathrm{C} 9$ & $110.39(13)$ \\
\hline $\mathrm{C} 6-\mathrm{C} 1-\mathrm{C} 8$ & $107.60(14)$ & $\mathrm{N} 2-\mathrm{C} 10-\mathrm{H} 10 \mathrm{~A}$ & 109.6 \\
\hline $\mathrm{O} 1-\mathrm{C} 2-\mathrm{C} 1$ & $117.25(15)$ & $\mathrm{N} 2-\mathrm{C} 10-\mathrm{H} 10 \mathrm{~B}$ & 109.6 \\
\hline $\mathrm{O} 1-\mathrm{C} 2-\mathrm{C} 3$ & $123.67(15)$ & $\mathrm{C} 9-\mathrm{C} 10-\mathrm{H} 10 \mathrm{~A}$ & 109.6 \\
\hline $\mathrm{C} 3-\mathrm{C} 2-\mathrm{C} 1$ & $119.08(16)$ & $\mathrm{C} 9-\mathrm{C} 10-\mathrm{H} 10 \mathrm{~B}$ & 109.6 \\
\hline $\mathrm{C} 2-\mathrm{C} 3-\mathrm{H} 3$ & 119.4 & $\mathrm{H} 10 \mathrm{~A}-\mathrm{C} 10-\mathrm{H} 10 \mathrm{~B}$ & 108.1 \\
\hline $\mathrm{C} 2-\mathrm{C} 3-\mathrm{C} 4$ & $121.20(17)$ & $\mathrm{N} 2-\mathrm{C} 11-\mathrm{H} 11 \mathrm{~A}$ & 109.5 \\
\hline $\mathrm{C} 4-\mathrm{C} 3-\mathrm{H} 3$ & 119.4 & $\mathrm{~N} 2-\mathrm{C} 11-\mathrm{H} 11 \mathrm{~B}$ & 109.5 \\
\hline $\mathrm{C} 3-\mathrm{C} 4-\mathrm{H} 4$ & 119.1 & $\mathrm{~N} 2-\mathrm{C} 11-\mathrm{H} 11 \mathrm{C}$ & 109.5 \\
\hline $\mathrm{C} 5-\mathrm{C} 4-\mathrm{C} 3$ & $121.82(18)$ & $\mathrm{H} 11 \mathrm{~A}-\mathrm{C} 11-\mathrm{H} 11 \mathrm{~B}$ & 109.5 \\
\hline $\mathrm{C} 5-\mathrm{C} 4-\mathrm{H} 4$ & 119.1 & $\mathrm{H} 11 \mathrm{~A}-\mathrm{C} 11-\mathrm{H} 11 \mathrm{C}$ & 109.5 \\
\hline $\mathrm{C} 4-\mathrm{C} 5-\mathrm{H} 5$ & 121.6 & $\mathrm{H} 11 \mathrm{~B}-\mathrm{C} 11-\mathrm{H} 11 \mathrm{C}$ & 109.5 \\
\hline $\mathrm{C} 4-\mathrm{C} 5-\mathrm{C} 6$ & $116.76(17)$ & $\mathrm{O} 2-\mathrm{C} 12-\mathrm{C} 13$ & $118.55(13)$ \\
\hline $\mathrm{C} 6-\mathrm{C} 5-\mathrm{H} 5$ & 121.6 & $\mathrm{O} 2-\mathrm{C} 12-\mathrm{O} 3$ & $124.96(14)$ \\
\hline $\mathrm{N} 1-\mathrm{C} 6-\mathrm{C} 1$ & $107.08(15)$ & $\mathrm{O} 3-\mathrm{C} 12-\mathrm{C} 13$ & $116.49(14)$ \\
\hline $\mathrm{N} 1-\mathrm{C} 6-\mathrm{C} 5$ & $129.66(16)$ & $\mathrm{C} 12-\mathrm{C} 13-\mathrm{H} 13$ & 117.6 \\
\hline $\mathrm{C} 5-\mathrm{C} 6-\mathrm{C} 1$ & $123.26(16)$ & $\mathrm{C} 133^{\mathrm{i}}-\mathrm{C} 13-\mathrm{C} 12$ & 124.77 (19) \\
\hline $\mathrm{N} 1-\mathrm{C} 7-\mathrm{H} 7$ & 124.7 & $\mathrm{C} 13^{\mathrm{i}}-\mathrm{C} 13-\mathrm{H} 13$ & 117.6 \\
\hline $\mathrm{O} 1-\mathrm{C} 2-\mathrm{C} 3-\mathrm{C} 4$ & $179.30(17)$ & $\mathrm{C} 6-\mathrm{C} 1-\mathrm{C} 2-\mathrm{C} 3$ & $1.4(2)$ \\
\hline $\mathrm{N} 1-\mathrm{C} 7-\mathrm{C} 8-\mathrm{C} 1$ & 0.79 (19) & $\mathrm{C} 6-\mathrm{C} 1-\mathrm{C} 8-\mathrm{C} 7$ & $-0.02(18)$ \\
\hline $\mathrm{N} 1-\mathrm{C} 7-\mathrm{C} 8-\mathrm{C} 9$ & $-178.45(16)$ & $\mathrm{C} 6-\mathrm{C} 1-\mathrm{C} 8-\mathrm{C} 9$ & $179.22(16)$ \\
\hline $\mathrm{C} 1-\mathrm{C} 2-\mathrm{C} 3-\mathrm{C} 4$ & $-0.7(3)$ & $\mathrm{C} 7-\mathrm{N} 1-\mathrm{C} 6-\mathrm{C} 1$ & $1.23(18)$ \\
\hline $\mathrm{C} 1-\mathrm{C} 8-\mathrm{C} 9-\mathrm{C} 10$ & $175.12(15)$ & $\mathrm{C} 7-\mathrm{N} 1-\mathrm{C} 6-\mathrm{C} 5$ & $-179.00(18)$ \\
\hline $\mathrm{C} 2-\mathrm{C} 1-\mathrm{C} 6-\mathrm{N} 1$ & $178.79(14)$ & $\mathrm{C} 7-\mathrm{C} 8-\mathrm{C} 9-\mathrm{C} 10$ & $-5.8(3)$ \\
\hline $\mathrm{C} 2-\mathrm{C} 1-\mathrm{C} 6-\mathrm{C} 5$ & $-1.0(2)$ & $\mathrm{C} 8-\mathrm{C} 1-\mathrm{C} 2-\mathrm{O} 1$ & $0.7(3)$ \\
\hline $\mathrm{C} 2-\mathrm{C} 1-\mathrm{C} 8-\mathrm{C} 7$ & $-179.45(18)$ & $\mathrm{C} 8-\mathrm{C} 1-\mathrm{C} 2-\mathrm{C} 3$ & $-179.26(18)$ \\
\hline $\mathrm{C} 2-\mathrm{C} 1-\mathrm{C} 8-\mathrm{C} 9$ & $-0.2(3)$ & $\mathrm{C} 8-\mathrm{C} 1-\mathrm{C} 6-\mathrm{N} 1$ & $-0.74(18)$ \\
\hline $\mathrm{C} 2-\mathrm{C} 3-\mathrm{C} 4-\mathrm{C} 5$ & $-0.5(3)$ & $\mathrm{C} 8-\mathrm{C} 1-\mathrm{C} 6-\mathrm{C} 5$ & $179.47(16)$ \\
\hline $\mathrm{C} 3-\mathrm{C} 4-\mathrm{C} 5-\mathrm{C} 6$ & $0.8(3)$ & $\mathrm{C} 8-\mathrm{C} 9-\mathrm{C} 10-\mathrm{N} 2$ & $178.34(14)$ \\
\hline $\mathrm{C} 4-\mathrm{C} 5-\mathrm{C} 6-\mathrm{N} 1$ & $-179.84(17)$ & $\mathrm{C} 11-\mathrm{N} 2-\mathrm{C} 10-\mathrm{C} 9$ & $178.49(15)$ \\
\hline $\mathrm{C} 4-\mathrm{C} 5-\mathrm{C} 6-\mathrm{C} 1$ & $-0.1(3)$ & $\mathrm{O} 2-\mathrm{C} 12-\mathrm{C} 13-\mathrm{C} 13^{\mathrm{i}}$ & $-13.3(3)$ \\
\hline $\mathrm{C} 6-\mathrm{N} 1-\mathrm{C} 7-\mathrm{C} 8$ & $-1.3(2)$ & $\mathrm{O} 3-\mathrm{C} 12-\mathrm{C} 13-\mathrm{C} 13^{\mathrm{i}}$ & $167.0(2)$ \\
\hline $\mathrm{C} 6-\mathrm{C} 1-\mathrm{C} 2-\mathrm{O} 1$ & $-178.63(14)$ & & \\
\hline
\end{tabular}

Symmetry code: (i) $-x+1,-y,-z+2$.

Hydrogen-bond geometry $\left(\AA,{ }^{\circ}\right)$

\begin{tabular}{lllll}
\hline$D-\mathrm{H} \cdots A$ & $D-\mathrm{H}$ & $\mathrm{H} \cdots A$ & $D \cdots A$ & $D-\mathrm{H} \cdots A$ \\
\hline $\mathrm{O} 1-\mathrm{H} 1 \cdots \mathrm{O} 3$ & $0.87(1)$ & $1.89(1)$ & $2.7399(16)$ & $163(2)$ \\
$\mathrm{N} 1-\mathrm{H} 1 A \cdots \mathrm{O} 2^{\mathrm{ii}}$ & $0.86(1)$ & $2.07(1)$ & $2.8854(18)$ & $157(2)$ \\
$\mathrm{N} 2-\mathrm{H} 2 A \cdots \mathrm{O} 3^{\mathrm{iii}}$ & $0.89(1)$ & $1.90(1)$ & $2.7349(18)$ & $155(2)$ \\
$\mathrm{N} 2-\mathrm{H} 2 B \cdots \mathrm{O} 2^{\text {iv }}$ & $0.89(1)$ & $1.91(1)$ & $2.7715(19)$ & $164(2)$
\end{tabular}

Symmetry codes: (ii) $x+1, y+1, z$; (iii) $x+1, y, z$; (iv) $-x+1,-y,-z+1$. 